\title{
Expectation of Pain Enhances Responses to Nonpainful Somatosensory Stimulation in the Anterior Cingulate Cortex and Parietal Operculum/Posterior Insula: an Event-Related Functional Magnetic Resonance Imaging Study
}

\author{
Nobukatsu Sawamoto, ${ }^{1}$ Manabu Honda, ${ }^{1,3}$ Tomohisa Okada, ${ }^{2,3}$ Takashi Hanakawa, ${ }^{1}$ Masutaro Kanda, ${ }^{1}$ \\ Hidenao Fukuyama, ${ }^{1}$ Junji Konishi, ${ }^{2}$ and Hiroshi Shibasaki ${ }^{1}$ \\ Departments of ${ }^{1}$ Brain Pathophysiology, Human Brain Research Center and ${ }^{2}$ Nuclear Medicine, Kyoto University Graduate \\ School of Medicine, Kyoto, 606-8507 Japan, and ${ }^{2}$ Laboratory of Cerebral Integration, National Institute for Physiological \\ Sciences, Okazaki, 444-8585 Japan
}

\begin{abstract}
Although behavioral studies suggest that pain distress may alter the perception of somatic stimulation, neural correlates underlying such alteration remain to be clarified. The present study was aimed to test the hypothesis that expectation of pain might amplify brain responses to somatosensory stimulation in the anterior cingulate cortex (ACC) and the region including parietal operculum and posterior insula (PO/PI), both of which may play roles in regulating pain-dependent behavior. We compared brain responses with and subjective evaluation of physically identical nonpainful warm stimuli between two psychologically different contexts: one linked with pain expectation by presenting the nonpainful stimuli randomly intermixed with painful stimuli and the other without. By applying the event-related functional magnetic resonance imaging technique, brain responses to the stimuli were assessed with respect to signal changes and activated
\end{abstract}

volume, setting regions of interest on activated clusters in ACC and bilateral PO/PI defined by painful stimuli. As a result, the uncertain expectation of painful stimulus enhanced transient brain responses to nonpainful stimulus in ACC and PO/PI. The enhanced responses were revealed as a higher intensity of signal change in ACC and larger volume of activated voxels in PO/PI. Behavioral measurements demonstrated that expectation of painful stimulus amplified perceived unpleasantness of innocuous stimulus. From these findings, it is suggested that ACC and $\mathrm{PO} / \mathrm{PI}$ are involved in modulation of affective aspect of sensory perception by the uncertain expectation of painful stimulus.

Key words: uncertain expectation of pain; innocuous stimulus; anterior cingulate cortex; parietal operculum; posterior insula; event-related functional magnetic resonance imaging
Because pain is characterized as an unpleasant and subjective experience (Merskey, 1986), its perception can be influenced by the psychophysical state of individuals (Cornwall and Donderi, 1988; Miyazaki et al., 1994; Rainville et al., 1997). Conversely, pain and associated distress can affect the biological state of individuals.

The effect of pain on an individual's state has been assessed from various viewpoints. Pain, and even just anxiety related to pain, were shown to cause diverse changes in the endocrine, immune, and other organ systems (Cousins, 1993; Herman and Cullinan, 1997). Functions of the CNS can also be modulated by personal experience of pain. Unpredictable shooting pain in neuralgia may induce severe distress even during the period without actual pain (Henderson, 1967). Severe pain can cause behavioral modulation, including increased sensitivity to external stimuli (Cousins, 1993). Pain distress often leads the sufferer to complain about nonspecific physical symptoms (McCracken et al., 1998). As the mechanism underlying the behavioral modulation by pain distress, the amplification of somatic sensation has been proposed (Barsky and Borus, 1999). However, neural correlates of the pain-induced CNS modulation remain to be clarified.

The anterior cingulate cortex (ACC) and the regions including

Received March 6, 2000; revised June 27, 2000; accepted July 17, 2000.

This work was supported in part by Grants-in-Aid for Scientific Research on Priority areas (C) 12210012 from the Japan Ministry of Education, Sciences, Sports, and Culture, Research for the Future Program JSPS-RFTF97L00201 from Japan Society for the Promotion Science, and a General Research grant for Aging and Health "Analysis of aged brain function with neuroimaging" from the Japan Ministry of Health and Welfare. We thank S. Nishizawa for technical support.

Correspondence should be addressed to Dr. Hiroshi Shibasaki, Department of Brain Pathophysiology, Human Brain Research Center, Kyoto University Graduate School of Medicine, 54 Kawahara-cho, Shogoin, Sakyo-ku, Kyoto, 606-8507 Japan. E-mail: shib@kuhp.kyoto-u.ac.jp.

Copyright (C) 2000 Society for Neuroscience $0270-6474 / 00 / 207438-08 \$ 15.00 / 0$ the parietal operculum and posterior insula $(\mathrm{PO} / \mathrm{PI})$ have been proposed as important cortical loci for pain perception based on human neuroimaging studies (Talbot et al., 1991; Casey et al., 1994, 1996; Coghill et al., 1994; Derbyshire et al., 1997; Xu et al., 1997; Becerra et al., 1999) and electrophysiological studies (Lenz et al., 1998a,b). The PO/PI is assumed to include the second somatosensory area (SII) (Penfield and Rasmussen, 1950; Penfield and Jasper, 1954). Single cell recordings have shown involvement of ACC and PO/PI not only in pain processing (Robinson and Burton, 1980; Sikes and Vogt, 1992) but also in mediating the diverse responses that can accompany pain (Dong et al., 1994; Koyama et al., 1998). Lesion of ACC or PO/PI can modify the emotional and behavioral reaction to pain, without impairing the ability to localize a painful stimulus (Foltz and White, 1968; Berthier et al., 1988). These findings suggest a role of ACC and $\mathrm{PO} / \mathrm{PI}$ in regulating the paindependent behavior.

This study was aimed to test the hypothesis that psychological distress related to pain might modulate neural responses to nonpainful somatosensory stimulation. On the basis of previous studies as discussed above, we focused on the responses in ACC and PO/PI among the multiple brain regions associated with pain processing. By applying the event-related functional magnetic resonance imaging (fMRI) technique, brain responses to and subjective evaluation of physically identical warm stimuli were compared between two psychologically different contexts. In one session, nonpainful warm stimuli were randomly intermixed with painful stimuli simulating unpredictable shooting pain like neuralgia. In the other session, only nonpainful stimuli were delivered. By comparing the responses with the nonpainful warm stimuli linked with and without expectation of painful stimulus, we explored the neural substrates involved in modulation of affective aspect of sensory perception by expectation of painful stimulus. 


\section{MATERIALS AND METHODS}

\section{Subjects}

Ten healthy male volunteers (aged 19-24 years, mean of 21.7 years) participated in this study. All were right-handed, and none had a previous history of any neurological or psychiatric disorders. Subjects had refrained from smoking and from consumption of alcohol or caffeine for a period of $24 \mathrm{hr}$ before the study. The protocol was approved by the Committee of Medical Ethics, Graduate School of Medicine and Faculty of Medicine, Kyoto University, and each subject gave written informed consent before the study.

\section{Stimulus and rating of subjective sensation}

We used a custom-made $\mathrm{CO}_{2}$ laser stimulator that delivered therma stimuli (Nippon Infrared Industries Co. Ltd., Kawasaki, Japan). The subjective sensation elicited by the stimulator was variable from warm to pain by adjusting the output power. The stimulus of $60 \mathrm{msec}$ duration was applied to the dorsum of the right hand. The diameter of the irradiation beam was adjusted to $\sim 6 \mathrm{~mm}$. To avoid habituation and sensitization, the stimulus spot was moved for every stimulus within the dorsum of the right hand. Detailed information on the $\mathrm{CO}_{2}$ laser stimuli was described previously (Miyazaki et al., 1994; Kanda et al., 1996a,b, 2000; Xu et al., 1997).

Before the imaging sessions, the output power corresponding to the pain threshold was determined individually by exposing the subject to stimuli of various intensity arranged in three increasing and decreasing series. The pain threshold was defined as the lowest stimulus intensity required for the subject to report a sensation as "just barely painful." Then, the subjects were asked to rate stimulus intensity and unpleasantness separately between 0 and 100 (Talbot et al., 1991; Casey et al., 1994, 1996; Derbyshire et al., 1997; Rainville et al., 1997; Svensson et al., 1997). In this scale, 0 indicated "no sensation" or "not at all unpleasant," and 100 indicated "the most intense pain imaginable" or "the most unpleasant feeling imaginable" for the intensity or unpleasantness, respectively. The intensity score of 70 was anchored to pain threshold. To explain the difference between intensity and unpleasantness to the subjects, standard instructions used by Price et al. (1989) were given as follows. "We are interested in two aspects of sensory experience. One is the intensity, that is, how strong the stimulus is felt. The other is unpleasantness, or how disturbing the stimulus is for you. The distinction between these two aspects of sensory experience might be made clearer if you think of listening to a sound, such as a radio. As the volume of the sound increases, I can ask you how loud it sounds, or how unpleasant it is to you. The intensity of the stimulus is like loudness, and the unpleasantness of the stimulus depends not only on intensity but also on other factors that may affect you. These are scales for measuring each of these two aspects of sensory experience. Although some sensory experiences may be equally intense and unpleasant, we would like you to judge these two aspects of your sensation independently." All subjects were trained to be able to rate sensation properly before the imaging sessions.

\section{Experimental conditions}

Each subject was examined by $\mathrm{CO}_{2}$ laser stimulation with two different intensities that were set to either 50-60\% [nonpainful warm stimulus (NPS)] or 160-200\% [painful stimulus (PS)] of the output power for each individual's pain threshold. These two radiant heat pulses generated by the $\mathrm{CO}_{2}$ laser stimulator were delivered in two psychologically different contexts that corresponded to two imaging sessions; one session was "uncertain," and the other was "certain." In the "uncertain context" session, 20 NPS and PS were presented in a randomized order, thus 40 stimuli in total. Subjects were told that two different stimuli, PS or NPS, would be given in a randomized order, but they were uncertain about which stimulus would be presented next. In the "certain context" session, only 20 NPS were delivered, and subjects were told beforehand that the PS would not be given in that particular session. Thus, the stimuli were categorized into three different conditions: PS in uncertain context (PS), NPS in uncertain context (NPS-u), and NPS in certain context (NPS-c).

PS may induce some changes on the stimulated part of skin, which can modulate the perception of NPS at that site (Duclaux and Kenshalo, 1980). To avoid such effects, the dorsal surface of the hand was divided into three areas, and each area was stimulated only in one particular condition. The location for the three conditions was randomized among the subjects. The order of uncertain and certain sessions was also random. Note that NPS-u and NPS-c were physically identical.

At $35.1 \mathrm{sec}$ after the beginning of each imaging trial that lasted $61.1 \mathrm{sec}$, a single stimulus was delivered. Subjects were told that the single stimulus would be presented within a fixed interval from the start of each trial. Thus, the subjects could predict the timing of the stimulus presentation in all the conditions but not the stimulus type in the uncertain condition. They were asked to remain silent and immobile during each trial and were required to report the ratings of each stimulus intensity and unpleasantness soon after each trial had ended. There was $\sim 30 \mathrm{sec}$ interval between successive trials to allow subjects to report the scores and to prepare for the next trial, resulting in the long interstimulus interval of $\sim 90 \mathrm{sec}$. The long prestimulus and poststimulus phases were used to obtain stable baseline, as well as to accurately evaluate the transient signal increase evoked by the stimulus. The long interstimulus interval was also useful to minimize the habituation effects and effectively induce anticipatory response.

\section{Image acquisition}

MRI scans were conducted using a whole-body 1.5 tesla scanner (Horizon; General Electric Medical Systems, Milwaukee, WI). Functional images were obtained with a $\mathrm{T}_{2} *$ sensitive, single-shot, echo-planar pulse sequence with the following parameters: repetition time (TR) $=1300 \mathrm{msec}$; echo time $(\mathrm{TE})=43 \mathrm{msec}$; flip angle $(\mathrm{FA})=60^{\circ}$; imaging matrix $=64 \times$ 64; field of view $=22 \times 22 \mathrm{~cm}$; slice thickness $=5 \mathrm{~mm}$; and slice gap $=1$ $\mathrm{mm}$. Eleven slices covering the ACC and PO/PI were acquired in an axial orientation. One imaging trial consisted of 47 functional scans (i.e., 61.1 sec). Subjects lay supine on an MRI scanner with their head immobilized by a forehead strap. Before the functional scans, a structural MRI of the whole brain was acquired using three-dimensional fast spoiled gradientrecalled at steady-state images ( $\mathrm{TR}=10.8 \mathrm{msec}, \mathrm{TE}=1.8 \mathrm{msec}$, inversion time $=300 \mathrm{msec}, \mathrm{FA}=15^{\circ}$, imaging matrix $=256 \times 256$, field of view $=$ $22 \times 22 \mathrm{~cm}$, slice thickness $=1.5 \mathrm{~mm}$, no slice gap, and 124 slices). Partial structural T1 weighted images corresponding to the area covered by echo-planar functional images were also obtained $(\mathrm{TR}=600 \mathrm{msec}, \mathrm{TE}=$ $17 \mathrm{msec}, \mathrm{FA}=30^{\circ}$, and imaging matrix $=256 \times 256$ ).

\section{Data analysis}

Image processing and statistical analysis. Image processing and statistical analysis were performed using SPM96 software (Wellcome Department of Cognitive Neurology, London, UK) with in-house modifications. Calculations and matrix manipulations were performed using Matlab (Mathworks, Natick, MA) on a Sun Sparc Ultra 2 workstation (Sun Microsystems, Mountain View, CA). The initial 12 scans (i.e., $15.6 \mathrm{sec}$ ) in each trial were excluded from the analysis because of the nonequilibrium state of magnetization, yielding 35 scans (i.e., $45.5 \mathrm{sec}$ ) to be analyzed in each trial. Of those 35 scans, the initial 15 scans corresponded to the prestimulus phase, the 16th scan to the stimulus phase, and the last 19 scans to the poststimulus phase. The effect of head motion was corrected by realigning all images to the first image using a least sum of squares method with threedimensional sinc interpolation (Friston et al., 1994). The activity in each voxel was linearly scaled with respect to the global activity. Data were smoothed in a spatial domain with a Gaussian filter (full width at half maximum $=5.16 \mathrm{~mm}$ ) to improve the signal-to-noise ratio.

The three different stimulus conditions (i.e., PS, NPS-u, and NPS-c) were analyzed separately using a general linear model (Friston et al., 1995a). Statistical analysis of fMRI time series data were conducted on an individual subject basis as described previously (Toma et al., 1999). To model the prestimulus, stimulus, and poststimulus phases, three boxcar functions were prepared. For each function, the value "1" was given for the phase of interest and " 0 " for the remaining phases. In the boxcar function for the stimulus phase, one scan time-locked to the stimulus was assigned to 1 . Each boxcar function was convolved with a Gaussian-shaped hemodynamic response function (delay, $5 \mathrm{sec}$; dispersion, $8 \mathrm{sec}$ ) (Friston et al., $1995 b$ ). Systematic difference across conditions was modeled as a confounding effect. The general linear model calculated a weighting coefficient for each regressor. We calculated $t$ deviates at each voxel by using a linear contrast of $[-1,2,-1]$ for [prestimulus, stimulus, poststimulus] phases to focus on a transient signal change associated with the stimulus. After transforming the $t$ value into $\mathrm{Z}$ scores with the unit normal distribution, the two-step analysis was conducted.

First step: analysis of anatomical location of activation. SPM $\{\mathrm{Z}\}$ maps consisting of the voxels with $\mathrm{Z}>3.09$ were created. Then, the correction for multiple comparisons was conducted by referring to the probabilistic behavior of Gaussian random fields. The threshold adopted was $p<0.05$. For the sake of convenience, the term "activation" in this study refers to the transient signal increases disclosed by the above analyses. In all subjects, PS produced activation clusters in the medial frontal lobe, consistent with ACC, and in the bilateral regions adjacent to the lateral sulcus, corresponding to PO/PI (Table 1). Similar activation was observed in nine subjects in NPS-u and seven subjects in NPS-c. To compare the location of activated areas among the subjects, SPM $\{\mathrm{Z}\}$ was transformed into the standardized Talairach space (Talairach and Tournoux, 1988) by applying the parameters obtained from the anatomical normalization of the wholebrain structural images after coregistering them with the mean functional images. Talairach coordinates of the activated voxels with maximum $\mathrm{Z}$ score were statistically compared among the three conditions using multivariate ANOVA (MANOVA). The analysis was conducted using the data from six subjects who showed significant activation in both ACC and bilateral PO/PI under all conditions. Note that the anatomically normalized data were used only for this purpose.

Second step: ROI setting and comparison of NPS- $u$ and NPS-c. To compare brain responses in the NPS-u and NPS-c conditions, the activation clusters identified in ACC and bilateral PO/PI under the PS condition in the first-step analysis were used as regions of interest (ROIs) in each individual subject (Fig. 1). Although pain and nonpainful warm sensations are mediated by different populations of sensory receptors in the skin and by different peripheral neuronal mechanisms, these two could evoke responses in the similar areas of the CNS, at least at the macroscopic level detected by the neuroimaging techniques (Becerra et al., 1999). In addition, because $\mathrm{CO}_{2}$ laser stimulus might induce thermal change on the skin during and after the radiation pulse, it appears reasonable to assume that spatial and temporal distribution of the change induced by the strong laser stimulus would include the skin volume in which the maximum tempera- 
Table 1. Mean coordinates of the statistical peak of activation in ACC and left and right PO/PI under PS, NPS-u, and NPS-c conditions (mean \pm SD)

\begin{tabular}{lcccr} 
Region/condition & $\mathrm{X}$ & $\mathrm{Y}$ & $\mathrm{Z}$ & $\begin{array}{r}\text { Number of } \\
\text { subjects* }\end{array}$ \\
\hline ACC & & & & \\
PS & $2.8 \pm 5.2$ & $15.0 \pm 8.3$ & $40.0 \pm 7.4$ & $10(10)$ \\
NPS-u & $3.0 \pm 6.2$ & $16.4 \pm 10.3$ & $41.4 \pm 6.7$ & $10(10)$ \\
NPS-c & $1.1 \pm 7.0$ & $17.5 \pm 5.8$ & $41.8 \pm 7.7$ & $8(10)$ \\
Left PO/PI & & & & \\
PS & $-56.2 \pm 7.7$ & $-17.6 \pm 3.9$ & $23.2 \pm 7.8$ & $10(10)$ \\
NPS-u & $-58.6 \pm 7.2$ & $-18.0 \pm 10.2$ & $25.8 \pm 10.0$ & $10(10)$ \\
NPS-c & $-59.5 \pm 6.6$ & $-15.0 \pm 7.6$ & $25.3 \pm 10.3$ & $8(10)$ \\
Right PO/PI & & & & $10(10)$ \\
PS & $59.8 \pm 6.4$ & $-17.4 \pm 7.0$ & $22.2 \pm 11.5$ & $9(10)$ \\
NPS-u & $57.6 \pm 7.9$ & $-10.4 \pm 7.4$ & $19.8 \pm 10.7$ & $7(9)$ \\
NPS-c & $57.3 \pm 14.0$ & $-12.3 \pm 8.3$ & $19.9 \pm 10.4$ & \\
\hline
\end{tabular}

*Activation corrected for multiple comparisons (activation uncorrected for multiple comparisons).

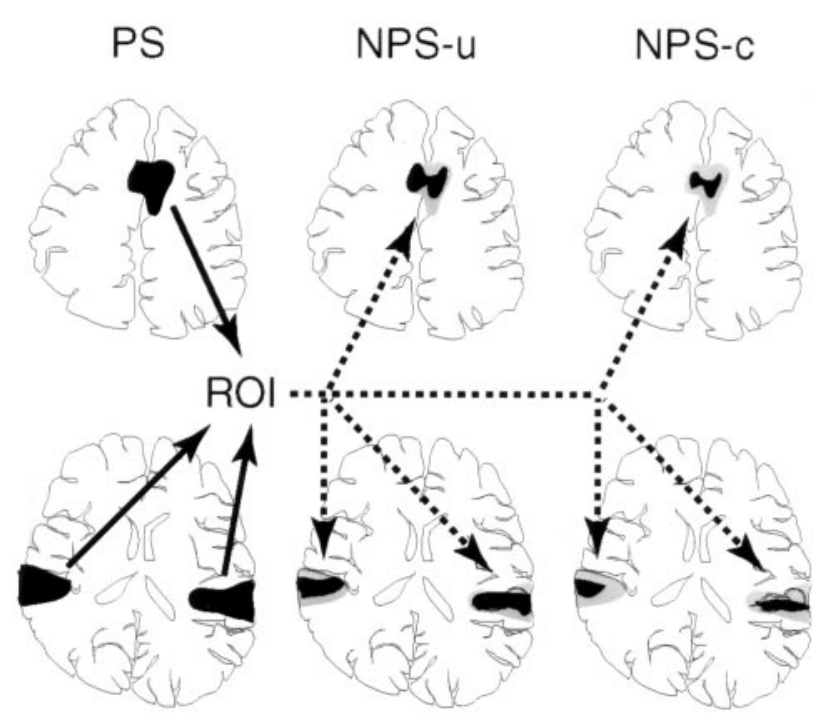

Figure 1. Schematic representation of the ROI setting in a representative individual subject. First, activated clusters under the painful stimulus (PS) condition were identified, and those clusters on the ACC and bilateral $\mathrm{PO} / \mathrm{PI}$ served as ROIs, which were then applied to the images of nonpainful warm stimulus in uncertain condition (NPS- $u$ ) and nonpainf ul warm stimulus in certain condition (NPS-c) in the same subject. Black areas indicate the actual activation in each condition, and gray areas represent ROIs identical to the activation in the PS condition. Note that ROIs are the same brain areas for NPS-u and NPS-c and are determined independently of these two conditions in each subject.

ture is below pain threshold but still enough to activate warmth receptors (Haimi-Cohen et al., 1983). Cerebral evoked potentials to painful laser stimulation seemed to include activation of warmth receptors (Towell et al., 1996). Taking these findings into account, the ROI determined by the PS were equally applied for the NPS-u and NPS-c. Note that the ROIs were determined independent of the activation by the NPS-u and NPS-c.

Brain activities in NPS-u and NPS-c were assessed with respect to both signal change and activated volume. For comparing signal change, first, the time course data of each voxel within ROI were averaged across 20 trials. Then, the time course data of each individual subject were averaged over voxels within ROI. We performed two kinds of signal change analyses regarding selection of voxels; in one analysis, all voxels within ROI were selected, whereas, in the other analysis, only the voxels above the predetermined threshold (i.e., $\mathrm{Z}>3.09$ ) in each condition were chosen. Note that the correction for multiple comparisons was not adopted for this purpose, partly because previous studies suggested the roles of ACC and $\mathrm{PO} / \mathrm{PI}$ in regulating pain-dependent behavior (Foltz and White, 1968; Berthier et al., 1988; Dong et al., 1994; Koyama et al., 1998). In addition, a broader criterion is appropriate to avoid false negative in selecting the data for further analysis (i.e., comparison of NPS-u and NPS-c). As shown in Figure 2, the averaged time course data revealed a gradual signal

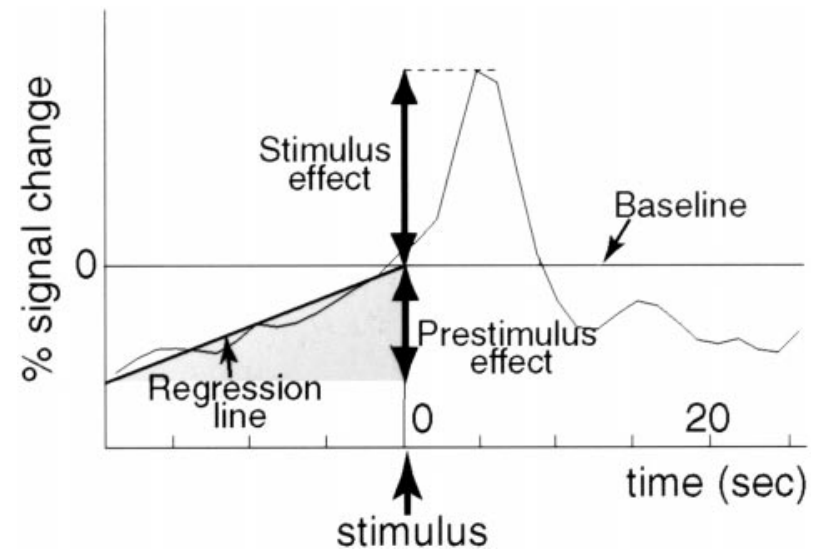

Figure 2. Schematic representation of the method used for evaluating the time course data of individual subjects. A regression line was calculated using the data during prestimulus phase under each condition for each subject. The intercept of the regression line at the time of the stimulus presentation served as the baseline signal intensity from which the signal change relating to the stimulus was divided into stimulus effect and prestimulus effect. The stimulus effect was assessed as the peak signal intensity, and the prestimulus effect was assessed as the signal intensity at the time of the first analyzed scan estimated from the regression line. Both effects were represented by the percent signal change with respect to the baseline (percent difference).

increase, even before the stimulus presentation. Thus, the regression line was calculated using the data of the prestimulus phase. The intercept of the regression line at time 0 (i.e., stimulus onset) was defined as the baseline signal intensity from which the signal change relating to the experimental condition was divided into two components: prestimulus effect and stimulus effect. The prestimulus effect was evaluated as the signal intensity at the time of the first analyzed scan within each condition estimated from the regression line, and the stimulus effect was assessed as the peak signal intensity after the stimulus onset. Both effects were represented by the percent signal change with respect to the baseline signal intensity (percent difference). Note that the prestimulus effects were negative values because the baseline was defined as the intensity at the time of the stimulus. The differences in prestimulus effects and stimulus effects between NPS-u and NPS-c conditions were statistically examined using paired $t$ tests. Signal change analysis of activated voxels in the right $\mathrm{PO} / \mathrm{PI}$ was conducted using data from nine subjects because of the lack of activation at the site in one subject. In the analyses of other ROIs, data from all subjects (i.e., 10 subjects) were used.

For comparing activated volume, the number of voxels above the threshold (i.e., $\mathrm{Z}>3.09$ ) in each condition was computed within each ROI and divided by the ROI voxel number. This can be interpreted as the ratio of activated volume in each condition with respect to those in the PS condition. A paired $t$ test was used to compare activated volumes between NPS-u and NPS-c conditions. 


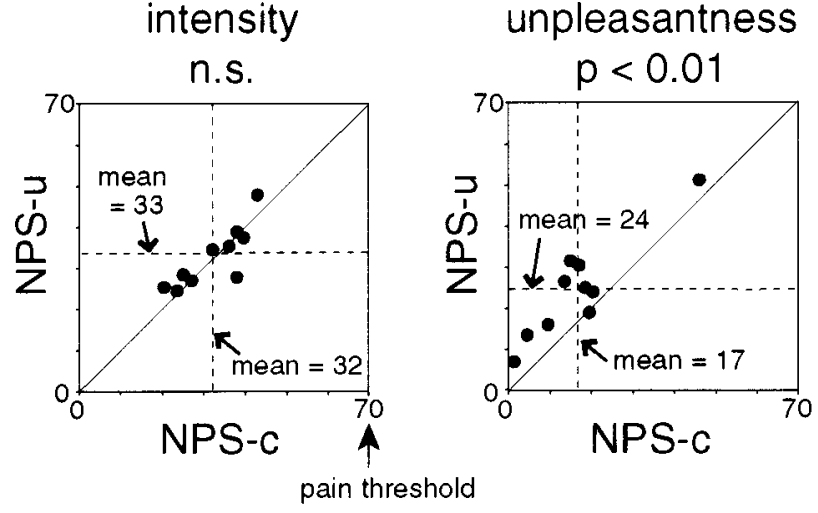

Figure 3. Subjective evaluation of nonpainful warm stimulus in uncertain condition (NPS-u) and nonpainful warm stimulus in certain condition (NPS-c). Scores of individual subjects and mean scores across all subjects are shown. Diagonal line indicates equal scores for NPS-u and NPS-c conditions. Subjects rated intensity and unpleasantness of each stimulus separately. The unpleasantness score of NPS-u was significantly higher than that of NPS-c, whereas the intensity scores did not show a significant difference between the two conditions. Statistical analysis was conducted with Wilcoxon signed rank tests.

\section{RESULTS}

\section{Stimulus intensity and subjective rating scores}

All subjects identified PS as a clearly painful stimulus and NPS as a stimulus below the pain threshold. PS was described as a distinct pricking sensation followed by a short-lasting burning aftersensation. NPS caused warm or very faint pricking sensation. The output power for the pain threshold, PS, and NPS was $5.2 \pm 0.9$ (mean \pm $\mathrm{SD}), 9.2 \pm 1.3$, and $2.9 \pm 0.6 \mathrm{~W}$, respectively.

In the subjective evaluation of intensity, PS was rated most intense, whereas NPS-u and NPS-c did not show significant differences from each other (Wilcoxon signed rank test; $p=0.31$ ) (Fig. $3)$. Subjective intensity scores were $82 \pm 7$ (mean \pm SD) for PS, $33 \pm 7$ for NPS-u, and $32 \pm 8$ for NPS-c conditions.

Subjective unpleasantness was dependent on the conditions. The highest score was obtained for PS. More importantly, NPS-u was reported to be more unpleasant than NPS-c (Wilcoxon signed rank test; $p<0.01$ ) (Fig. 3). Unpleasantness scores were $69 \pm 15$ for PS, $24 \pm 12$ for NPS-u, and $17 \pm 12$ for NPS-c. The observation of positive unpleasantness scores in response to completely nonpainful stimulus seems reasonable, because the unpleasantness was not necessarily associated with pain depending on the instruction (Price et al., 1989; Svensson et al., 1997).

The unpleasantness scores of all the subjects were actually below 70 in NPS-u and NPS-c conditions, although they could have varied as much as 100 points. The grand averaged unpleasantness scores were smaller than the intensity scores. Therefore, it is reasonable to conclude that the present finding is not attributable to an artifact introduced by the different scaling range of the scores.

\section{Activated areas in each condition}

PS evoked activation in the medial frontal lobe (i.e., ACC) and the regions adjacent to the lateral sulcus (i.e., $\mathrm{PO} / \mathrm{PI}$ ) in all subjects. Figure $4 \mathrm{~A}$ indicates brain responses of a representative subject superimposed on his own anatomical MRI. The activated clusters in the PS condition served as the ROI for ACC and bilateral PO/PI for each individual subject. Volumes of activated clusters were $5.67 \pm 3.95 \mathrm{ml}$ (mean $\pm \mathrm{SD}$ ) in ACC, $7.76 \pm 4.23 \mathrm{ml}$ in the left $\mathrm{PO} / \mathrm{PI}$, and $10.27 \pm 6.51 \mathrm{ml}$ in the right $\mathrm{PO} / \mathrm{PI}$.

NPS-u and NPS-c produced brain activation in regions similar to those by PS, as shown in Figure $4 A$. In both conditions, the activity in ACC and bilateral PO/PI was observed in almost all subjects (nine subjects in NPS-u and seven subjects in NPS-c) (Table 1). MANOVA indicated no significant difference in the location of the peak activation among PS, NPS-u, and NPS-c in ACC (Wilks'

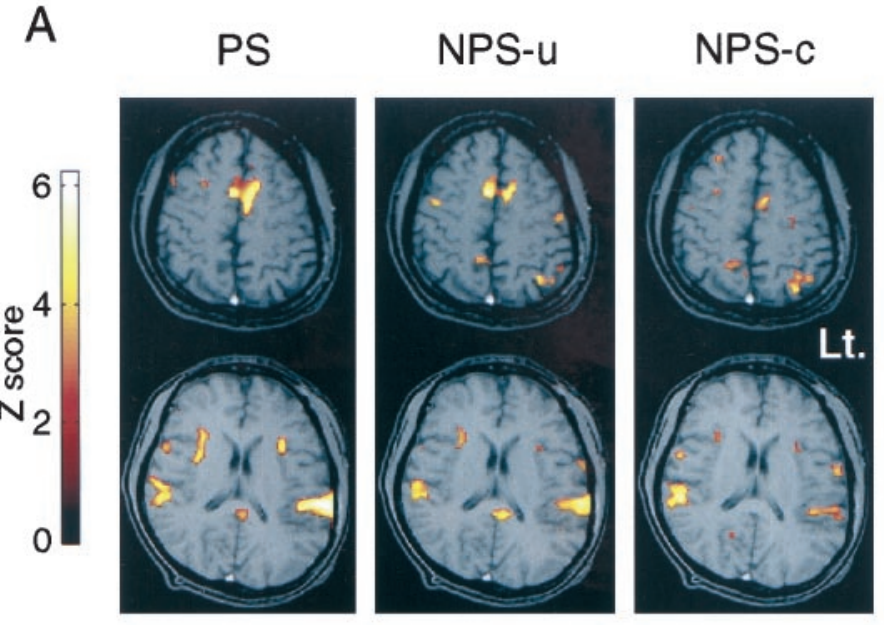

B

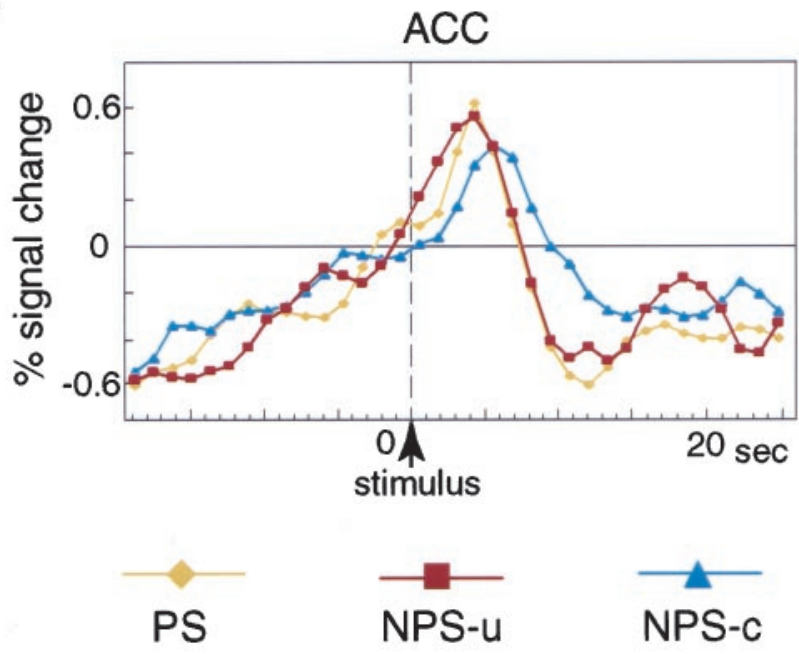

Figure 4. Activated areas for PS, NPS-u, and NPS-c conditions $(A)$ and averaged time course data of activated cluster in ACC for each condition $(B)$, obtained from a single subject. $A$, Activated areas, which showed significant transient signal increase time-locked to the stimulus, are superimposed on the subject's own structural MRI. Activation is seen in ACC, bilateral PO/PI, anterior insula, and other areas. The activated areas look similar in PS, NPS-u, and NPS-c conditions. The right side of the brain is shown on the left side of the image. Brighter color represents a higher statistical significance. $B$, Averaged signals across 20 trials at the activated cluster in ACC are shown for PS, NPS-u, and NPS-c conditions. The vertical dotted line indicates the time of the stimulus presentation. Transient signal increase after the stimulus (stimulus effect) and gradual signal increase before the stimulus (prestimulus effect) are observed. The stimulus effect is highest in PS and higher in NPS-u compared with NPS-c condition.

lambda $=0.78 ; p=0.75$ ), left PO/PI (Wilks' lambda $=0.69 ; p=$ 0.52 ), or right $\mathrm{PO} / \mathrm{PI}$ (Wilks' lambda $=0.72 ; p=0.60$ ). The Talairach coordinates of activated areas by PS and NPS were consistent with the previous studies (Talbot et al., 1991; Coghill et al., 1994; Xu et al., 1997; Becerra et al., 1999). Activation by PS, NPS-u, and NPS-c was also observed in other multiple brain areas including, the anterior insula, thalamus, and prefrontal and premotor cortices.

Averaged time course data of the activated cluster on ACC in a representative subject are shown in Figure $4 B$. As expected, the stimulus effect of PS was higher than NPS conditions. More importantly, the stimulus effect of NPS-u was greater than that of NPS-c. Time course data of individual subjects revealed that the latencies of peak signal response to each stimulus were shorter than $7.8 \mathrm{sec}$ after the stimulus presentation. 

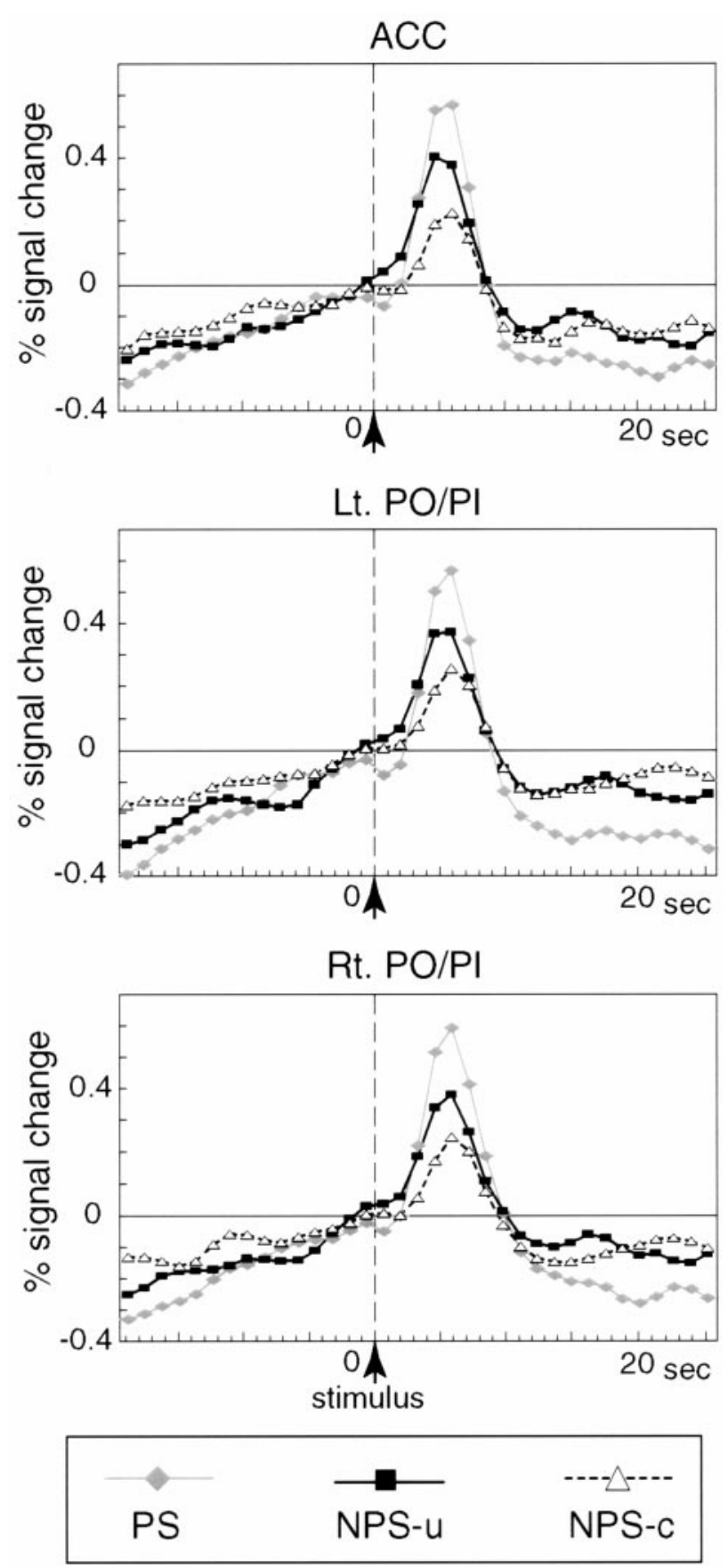

Figure 5. Averaged time course data of all voxels within each ROI. Signal changes in ACC and left and right PO/PI under each of the PS, NPS-u, and NPS-c conditions were averaged across all subjects. The vertical dotted line indicates the time of the stimulus presentation. Transient signal increase after the stimulus (stimulus effect) and gradual signal increase before the stimulus (prestimulus effect) are observed in all ROIs under all conditions. In all ROIs, the stimulus effects are highest in PS and higher in NPS-u compared with NPS-c condition.

\section{Comparison of activation between NPS-u and NPS-c}

NPS-u and NPS-c were compared using the voxels within the ROIs. Figure 5 represents the time course data of all voxels within each ROI averaged across all subjects, comparing PS, NPS-u, and NPS-c conditions. A gradual signal increase before the stimulus (prestimulus effect) and a transient signal change after the stimulus (stimulus effect) were observed in all ROIs under all conditions.

Results of signal change analyses are presented in Figure 6, $A$ and $B$, and Table 2 . The prestimulus effects showed no significant differences between NPS-u and NPS-c in any of the three ROIs, in

\section{A. Signal change of all voxels (stimulus effect)}

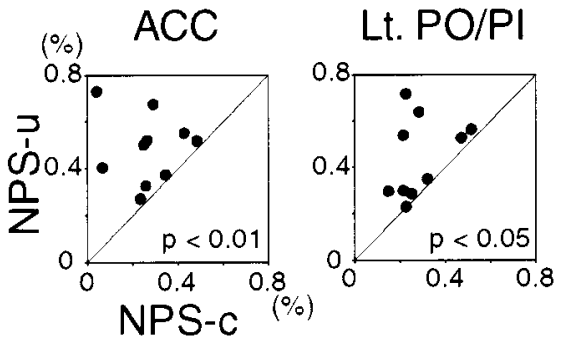

Rt. PO/PI

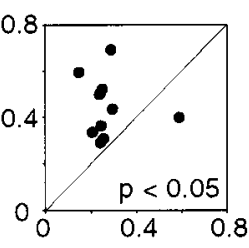

B. Signal change of selected voxels

(stimulus effect)
ACC

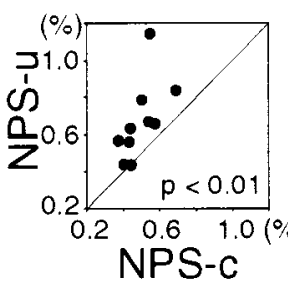

C. Activated volume

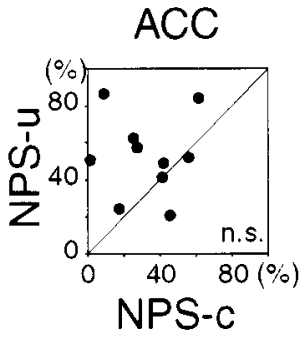

Lt. PO/PI

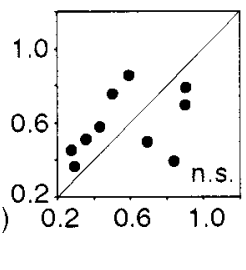

Rt. $\mathrm{PO} / \mathrm{PI}$

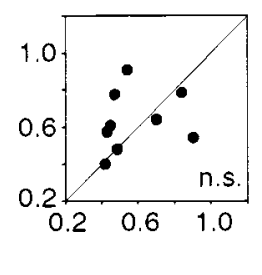

Figure 6. Comparison of NPS-u with NPS-c conditions. Each dot represents individual subject data. Diagonal line indicates equal responses in NPS-u and NPS-c conditions. The two conditions are compared with respect to signal change and activated volume setting ROI on ACC and bilateral PO/PI. For signal change comparison, the time course data of each subject are averaged over voxels within ROI: in one analysis averaged over all voxels $(A)$ and in the other analysis averaged over selected voxels in each condition (i.e., $\mathrm{Z}>3.09)(B)$. Transient signal changes after the stimulus (stimulus effect; see Fig. 2) are shown. For comparing activated volume, the number of activated voxels above the threshold (i.e., $\mathrm{Z}>3.09$ ) in each condition was computed within each ROI and divided by the ROI voxel number $(C)$. As for the signal change of all voxels $(A)$, stimulus effect averaged over all voxels within ROI revealed significantly larger change in NPS-u than NPS-c condition in ACC and left and right PO/PI. As for the signal change of selected voxels $(B)$, stimulus effect averaged over the selected voxels within ROI revealed significantly larger change in NPS-u than in NPS-c condition in ACC. In contrast, the response in bilateral $\mathrm{PO} / \mathrm{PI}$ was not different between the two conditions. In regards to the activated volume $(C)$, the proportions of the activated volume in NPS-u were significantly larger than NPS-c in bilateral PO/PI. In contrast, the difference in ACC did not reach statistical significance. In summary, higher intensity of signal change after the stimulus in ACC and larger volume of activated voxels in bilateral $\mathrm{PO} / \mathrm{PI}$ are consistently observed, which suggests enhanced brain responses in NPS-u compared with NPS-c. Statistical analyses were conducted using paired $t$ tests.

either all voxels or selected voxels comparisons (i.e., $Z>3.09$ ). In ACC and bilateral PO/PI, the stimulus effects of all voxels in NPS-u were significantly higher than those in NPS-c. Regarding the selected voxels within ROI, the stimulus effect was higher in NPS-u than in NPS-c only in ACC, whereas the effects of the two conditions were not different in bilateral PO/PI. Results of activated 
Table 2. Comparison between NPS-u and NPS-c (mean \pm SD)

\begin{tabular}{|c|c|c|c|c|c|}
\hline \multirow[b]{2}{*}{ Region/condition } & \multicolumn{2}{|c|}{ Signal change of ROI } & \multicolumn{2}{|c|}{ Signal change of selected voxels } & \multirow{2}{*}{$\frac{\text { Activated volume }}{\text { Volume (\%) }}$} \\
\hline & Prestimulus effect (\%) & Stimulus effect $(\%)$ & Prestimulus effect $(\%)$ & Stimulus effect $(\%)$ & \\
\hline \multicolumn{6}{|l|}{$\mathrm{ACC}$} \\
\hline NPS-u & $-0.21 \pm 0.19$ & $0.49 \pm 0.15$ & $-0.28 \pm 0.18$ & $0.67 \pm 0.21$ & $53 \pm 22$ \\
\hline NPS-c & $-0.15 \pm 0.24$ & $0.27 \pm 0.14$ & $-0.21 \pm 0.20$ & $0.49 \pm 0.10$ & $33 \pm 20$ \\
\hline$p$ & 0.38 & $<0.01$ & 0.25 & $<0.01$ & 0.059 \\
\hline \multicolumn{6}{|l|}{ Left PO/PI } \\
\hline NPS-u & $-0.27 \pm 0.18$ & $0.45 \pm 0.17$ & $-0.32 \pm 0.20$ & $0.59 \pm 0.17$ & $55 \pm 23$ \\
\hline NPS-c & $-0.16 \pm 0.18$ & $0.29 \pm 0.12$ & $-0.25 \pm 0.28$ & $0.58 \pm 0.19$ & $29 \pm 16$ \\
\hline$p$ & 0.08 & $<0.05$ & 0.38 & 0.89 & $<0.005$ \\
\hline \multicolumn{6}{|l|}{ Right $\mathrm{PO} / \mathrm{PI}$} \\
\hline NPS-u & $-0.23 \pm 0.23$ & $0.45 \pm 0.13$ & $-0.32 \pm 0.21$ & $0.62 \pm 0.16$ & $46 \pm 18$ \\
\hline NPS-c & $-0.13 \pm 0.14$ & $0.28 \pm 0.12$ & $-0.21 \pm 0.20$ & $0.58 \pm 0.19$ & $30 \pm 22$ \\
\hline$p$ & 0.34 & $<0.05$ & 0.27 & 0.48 & $<0.05$ \\
\hline
\end{tabular}

$p$, Statistical analysis using the paired $t$ test.

volume analyses (i.e., $\mathrm{Z}>3.09$ ) are shown in Figure $6 C$ and Table 2. In bilateral PO/PI, the activated volumes in the NPS-u condition were significantly greater than those in NPS-c. In contrast, in ACC, the volume showed a larger tendency in NPS-u than NPS-c, but the difference did not reach statistical significance. In summary, higher intensity of signal change after the stimulus in ACC and larger volume of activated volume in PO/PI were consistently shown, which suggests enhanced brain responses in NPS-u compared with NPS-c. The relationship between the increase of signal intensity and activation volume might be interpreted by the models of Baker et al. (1999) in which fMRI signal comprises a Gaussian distribution.

\section{DISCUSSION}

The present fMRI study showed that, in normal human subjects, the uncertain expectation of painful stimulation enhanced the transient brain responses to nonpainful warm stimulation in the $\mathrm{ACC}$ and PO/PI. In the behavioral measurements, the expectation of painful stimulation amplified perceived unpleasantness of even innocuous stimulation, whereas it did not influence the perceived intensity. From these findings, ACC and PO/PI are considered to be involved in modulation of affective aspect of sensory perception by the uncertain expectation of painful stimulus.

In pain studies using persistent or trains of painful stimuli, it has been hypothesized that pain distress might enhance the stimulusevoked neural responses (Coghill et al., 1994). This hypothesis was tested in the present study by comparing the responses to nonpainful warm stimuli linked with and without pain expectation. To link the warm stimulus with pain expectation, we randomly presented nonpainful stimuli intermixed with painful stimuli. This kind of paradigm design can be effectively analyzed by event-related procedures (Rosen et al., 1998). The procedure also enabled us to assess how subjects felt the stimulus on a trial-by-trial basis. In addition, within-trial responses were chronologically separated into two components: the response before the stimulus (prestimulus effect) and the response after the stimulus (stimulus effect). Comparison of fMRI responses to nonpainf ul warm stimuli between the two psychologically different contexts showed that only the stimulus effect, but not the prestimulus effect, was significantly enhanced by the uncertain expectation of painful stimulus. Thus, the enhancement may not be caused just by the induced psychological state but by the interaction of the psychological state with the sensory processes.

The response to somatic stimuli in the CNS can be exaggerated in patients suffering from pain (Dahl et al., 1992; Flor et al., 1997). However, this enhancement may be associated with complex features of pain, which could be affected by tissue injury, psychological aspects, and other factors. Among these effects, the peripheral responses related to pain were unlikely to cause the enhancement in the present study. Pain may induce some changes on the skin, which can modulate somatic sensation (Duclaux and Kenshalo, 1980). To avoid such effects, three different areas were prepared for painful stimulus, nonpainful stimulus with pain expectation, and nonpainful stimulus without pain expectation, and the locations of these areas were randomized among the subjects. Therefore, psychological distress related to pain could have been the main cause of the enhanced cortical activity.

Anticipation of pain with uncertainty would play a substantial role in psychological modulation. In a previous behavioral study, uncertain pain was shown to increase unpleasantness and to result in less pain tolerance compared with certain pain (Staub et al., 1971). The present findings may support the behavioral study, although we investigated the response to nonpainful stimulus instead of painful stimulus. In contrast, uncertainty about sensory stimuli, regardless of their properties, modulates brain responses and evokes potentials of positive deflection at $300 \mathrm{msec}$ after the stimulus (i.e., P300) that are recorded from widespread brain areas (Sutton et al., 1965; McCarthy et al., 1997). This nonspecific uncertainty effect should also be considered as a component of the modulatory factors.

Selective attention to the stimulus modulates the activity of early sensory processing areas (Frith and Dolan, 1997; Mima et al., 1998). Although the present findings did not contradict those previous studies, it is unlikely that the psychological modulation documented in the present study would be exactly the same as the effect of selective attention itself, because subjects had to attend to and evaluate the stimulus regardless of the presence or absence of pain anticipation.

The sensations of pain and cold are mediated by parallel ascending sensory channels that interact with each other, and these interactions can influence brain responses to thermal stimulus (Craig and Bushnell, 1994; Craig et al., 1996). Although cold and warm sensations are mediated by separate populations of receptors and by different neural mechanisms (Darian-Smith, 1984), the interaction between the systems that mediate pain and warmth might also have affected brain responses to warm stimuli adopted in the present study (Duncan et al., 1998).

\section{Role of ACC in modulation of affective aspect of sensory perception by pain expectation}

ACC is an important area for processing sensory information related to pain (Devinsky et al., 1995). The fact that neural responses to pain in ACC have almost no localizing information suggests its role in affective coding (Sikes and Vogt, 1992). Neuronal recordings and neuroimaging studies suggest that ACC is involved in mediating the affective components associated not only with painful stimulation but also with attention and anticipation of upcoming painful stimulation (Koyama et al., 1998; Hsieh et al., 
1999; Hutchison et al., 1999; Ploghaus et al., 1999). These neural properties are consistent with the present findings that expectation of pain modulates ACC responses. The present findings may also agree with clinical observations of pain in patients treated by limbic surgery. Those patients, who are too precipitously reactive to their environment and showed augmented pain symptoms, appeared to be relieved by cingulotomy and no longer suffer from those symptoms after cingulotomy, although they recognize chronic pain as it had been (Foltz and White, 1968). This observation suggests an important role of ACC in regulating pain-dependent behavior.

On the other hand, in the present study, the ACC response to innocuous stimulus also increased in association with the enhancement of the unpleasantness. The parallel relationship between pain unpleasantness and the ACC responses was elucidated in a positron emission tomography study (Rainville et al., 1997). These findings of Rainville et al. suggested the significant involvement of ACC in the affective component of pain. The present study may extend their view and implicate the involvement of ACC in processing negative affect associated with somatic stimulation independent of pain sensation. Rainville et al. (1997) demonstrated a significantly increased signal change and activation volume in ACC in relation to increased unpleasantness. Although the fMRI signal of ACC significantly increased in the present study, the increase in activated volume in ACC did not reach statistical significance. The difference might be attributable to the low spatial resolution of positron emission tomography or intersubject spatial averaging of images for group analysis, which was not used in the present study (Sadato et al., 1997)

Overlapping activation and constant anticipatory responses of ACC provoked by noxious and innocuous stimulation even without pain anticipation may reflect nonspecific function, such as general arousal effect (Vogt and Sikes, 2000). The activation could be associated with a broad role of ACC in modulating behavioral reactions to external stimuli (Devinsky et al., 1995).

\section{Role of PO/PI in modulation of affective aspect of sensory perception by pain expectation}

Neuronal recordings from monkey PO/PI have identified nociceptive neurons in the SII, neighboring area $7 \mathrm{~b}$, and posterior insula (Robinson and Burton, 1980). The response characteristics of nociceptive neurons in SII and area $7 \mathrm{~b}$ suggest an involvement of those neurons in learning and attention to events that produce pain (Dong et al., 1994; Treede et al., 1999). Anatomical evidence suggests that the route from the parietal operculum to posterior insula may act as a principal neural relay for conveying somatosensory information into the limbic system and thus provide a means for interrelating the painful events with relevant affective states (Mesulam and Mufson, 1982). Moreover, the lesion in PO/PI may produce a clinical syndrome called asymbolia for pain in which patients recognize pain but lack appropriate affective responses to painful stimulation (Berthier et al., 1988). The evidence may concur with the present findings implicating functional modulation of $\mathrm{PO} / \mathrm{PI}$ by expectation of pain. A subdivision of the somatosensory areas around the operculum was proposed (Krubitzer et al., 1986; Mima et al., 1997). The greater volume of activation caused by expectation of pain might be associated with multiple sensory representation within the operculum.

In summary, the neuroimaging method in the present study had advantages for assessing the functional neuroanatomy of pain distress. The event-related experimental design allowed us to use random-order presentation of different stimuli, to assess subjective evaluation of the stimuli on a trial-by-trial basis, and to analyze transient brain responses. The present findings suggest that the ACC and PO/PI are involved in modulation of affective aspect of sensory perception by the uncertain expectation of painful stimulus. Interpreting these findings in the light of psychological and biological meaning of pain, the modulation might be considered as an adaptive response to pain as a warning signal. However, when pain is excessively severe or prolonged, the response may lead to deleterious effects. Understanding this aspect of pain from a view- point of symptomatic treatment may contribute to the control of the complex nature of pain experiences.

\section{REFERENCES}

Baker JT, Donoghue JP, Sanes JN (1999) Gaze direction modulates finger movement activation patterns in human cerebral cortex. J Neurosci 19:10044-10052.

Barsky AJ, Borus JF (1999) Functional somatic syndromes. Ann Intern Med 130:910-921.

Becerra LR, Breiter HC, Stojanovic M, Fishman S, Edwards A, Comite AR, Gonzalez RG, Borsook D (1999) Human brain activation under controlled thermal stimulation and habituation to noxious heat: an fMRI study. Magn Reson Med 41:1044-1057.

Berthier M, Starkstein S, Leiguarda R (1988) Asymbolia for pain: a sensory-limbic disconnection syndrome. Ann Neurol 24:41-49.

Casey KL, Minoshima S, Berger KL, Koeppe RA, Morrow TJ, Frey KA (1994) Positron emission tomographic analysis of cerebral structures activated specifically by repetitive noxious heat stimuli. J Neurophysiol $71: 802-807$.

Casey KL, Minoshima S, Morrow TJ, Koeppe RA (1996) Comparison of human cerebral activation pattern during cutaneous warmth, heat pain, and deep cold pain. J Neurophysiol 76:571-581.

Coghill RC, Talbot JD, Evans AC, Meyer E, Gjedde A, Bushnell MC, Duncan GH (1994) Distributed processing of pain and vibration by the human brain. J Neurosci 14:4095-4108.

Cornwall A, Donderi DC (1988) The effect of experimentally induced anxiety on the experience of pressure pain. Pain 35:105-113.

Cousins M (1993) Acute and postoperative pain. In: Textbook of pain (Wall PD, Melzack R, eds), pp 357-385. Edinburgh: Churchill Livingstone.

Craig AD, Bushnell MC (1994) The thermal grill illusion: unmasking the burn of cold pain. Science 265:252-255.

Craig AD, Reiman EM, Evans A, Bushnell MC (1996) Functional imaging of an illusion of pain. Nature 384:258-260.

Dahl JB, Erichsen CJ, Fuglsang-Frederiksen A, Kehlet H (1992) Pain sensation and nociceptive reflex excitability in surgical patients and human volunteers. Br J Anaesth 69:117-121.

Darian-Smith I (1984) Thermal sensibility. In: Handbook of physiology, Sec 1, The nervous system, Vol III, Sensory processes, Pt 2 (Darian-Smith I, ed), pp 879-913. Bethesda, MD: American Physiological Society.

Derbyshire SW, Derbyshire SW, Jones AK, Gyulai F, Clark S, Townsend D, Firestone LL (1997) Pain processing during three levels of noxious stimulation produces differential patterns of central activity. Pain 73:431-445.

Devinsky O, Morrell MJ, Vogt BA (1995) Contributions of anterior cingulate cortex to behaviour. Brain 118:279-306.

Dong WK, Chudler EH, Sugiyama K, Roberts VJ, Hayashi T (1994) Somatosensory, multisensory, and task-related neurons in cortical area $7 \mathrm{~b}(\mathrm{PF})$ of unanesthetized monkeys. J Neurophysiol 72:542-564.

Duclaux R, Kenshalo Sr DR (1980) Response characteristics of cutaneous warm receptors in the monkey. J Neurophysiol 43:1-15.

Duncan GH, Kupers RC, Marchand S, Villemure JG, Gybels JM, Bushnell MC (1998) Stimulation of human thalamus for pain relief: possible modulatory circuits revealed by positron emission tomography. J Neurophysiol 80:3326-3330.

Flor H, Braun C, Elbert T, Birbaumer N (1997) Extensive reorganization of primary somatosensory cortex in chronic back pain patients. Neurosci Lett 224:5-8.

Foltz EL, White LE (1968) The role of rostral cingulumotomy in "pain" relief. Int J Neurol 6:353-373.

Friston KJ, Jezzard P, Turner R (1994) Analysis of functional MRI timeseries. Hum Brain Mapp 1:153-171.

Friston KJ, Holmes AP, Worsley KJ, Poline JB, Frith C, Frackowiak RS (1995a) Statistical parametric maps in functional imaging: a general linear approach. Hum Brain Mapp 2:189-210.

Friston KJ, Holmes AP, Poline JB, Grasby PJ, Williams SC, Frackowiak RS, Turner R (1995b) Analysis of fMRI time-series revisited. NeuroImage 2:45-53.

Frith C, Dolan RJ (1997) Brain mechanisms associated with top-down processes in perception. Philos Trans R Soc Lond B Biol Sci 352:1221-1230.

Haimi-Cohen R, Cohen A, Carmon A (1983) A model for the temperature distribution in skin noxiously stimulated by a brief pulse of $\mathrm{CO} 2$ laser radiation. J Neurosci Methods 8:127-137.

Henderson WR (1967) Trigeminal neuralgia: the pain and its treatment. Br Med J 1:7-15.

Herman JP, Cullinan WE (1997) Neurocircuitry of stress: central control of the hypothalamo-pituitary-adrenocortical axis. Trends Neurosci 20:78-84. Hsieh JC, Stone-Elander S, Ingvar M (1999) Anticipatory coping of pain expressed in the human anterior cingulate cortex: a positron emission tomography study. Neurosci Lett 262:61-64.

Hutchison WD, Davis KD, Lozano AM, Tasker RR, Dostrovsky JO (1999) Pain-related neurons in the human cingulate cortex. Nat Neurosci 2:403-405.

Kanda M, Fujiwara N, Xu X, Shindo K, Nagamine T, Ikeda A, Shibasaki $\mathrm{H}$ (1996a) Pain-related and cognitive components of somatosensory evoked potentials following $\mathrm{CO} 2$ laser stimulation in man. Electroencephalogr Clin Neurophysiol 100:105-114. 
Kanda M, Mima T, Xu X, Fujiwara N, Shindo K, Nagamine T, Ikeda A, Shibasaki H (1996b) Pain-related somatosensory evoked potentials can quantitatively evaluate hypalgesia in Wallenberg's syndrome. Acta Neurol Scand 94:131-136.

Kanda M, Nagamine T, Ikeda A, Ohara S, Kunieda T, Fujiwara N, Yazawa S, Sawamoto N, Matumoto R, Taki W, Shibasaki H (2000) Primary somatosensory cortex is actively involved in pain processing in human. Brain Res 853:282-289.

Koyama T, Tanaka YZ, Mikami A (1998) Nociceptive neurons in the macaque anterior cingulate activate during anticipation of pain. NeuroReport 9:2663-2667.

Krubitzer LA, Sesma MA, Kaas JH (1986) Microelectrode maps, myeloarchitecture, and cortical connections of three somatotopically organized representations of the body surface in the parietal cortex of squirrels. J Comp Neurol 250:403-430.

Lenz FA, Rios M, Zirh A, Chau D, Krauss G, Lesser RP (1998a) Painful stimuli evoke potentials recorded over the human anterior cingulate gyrus. J Neurophysiol 79:2231-2234.

Lenz FA, Rios M, Chau D, Krauss GL, Zirh TA, Lesser RP (1998b) Painful stimuli evoke potentials recorded from the parasylvian cortex in humans. J Neurophysiol 80:2077-2088.

McCarthy G, Luby M, Gore J, Goldman-Rakic P (1997) Infrequent events transiently activate human prefrontal and parietal cortex as measured by functional MRI. J Neurophysiol 77:1630-1634.

McCracken LM, Faber SD, Janeck AS (1998) Pain-related anxiety predicts non-specific physical complaints in persons with chronic pain. Behav Res Ther 36:621-630.

Merskey H (1986) Classification of chronic pain. Descriptions of chronic pain syndromes and definitions of pain terms. Prepared by the International Association for the Study of Pain, Subcommittee on Taxonomy. Pain [Suppl] 3:S1-S226.

Mesulam MM, Mufson EJ (1982) Insula of the old world monkey. III. Efferent cortical output and comments on function. J Comp Neurol 212:38-52.

Mima T, Ikeda A, Nagamine T, Yazawa S, Kunieda T, Mikuni N, Taki W, Kimura J, Shibasaki H (1997) Human second somatosensory area: subdural and magnetoencephalographic recording of somatosensory evoked responses. J Neurol Neurosurg Psychiatry 63:501-505.

Mima T, Nagamine T, Nakamura K, Shibasaki H (1998) Attention modulates both primary and second somatosensory cortical activities in humans: a magnetoencephalographic study. J Neurophysiol 80:2215-2221.

Miyazaki M, Shibasaki H, Kanda M, Xu X, Shindo K, Honda M, Ikeda A, Nagamine T, Kaji R, Kimura J (1994) Generator mechanism of painrelated evoked potentials following $\mathrm{CO} 2$ laser stimulation of the hand: scalp topography and effect of predictive warning signal. J Clin Neurophysiol 11:242-254.

Penfield W, Jasper H (1954). Functional localization in the cerebral cortex. In: Epilepsy and the functional anatomy of the human brain, pp 41-155. Boston: Little Brown.

Penfield W, Rasmussen T (1950) Secondary sensory and motor representation. In: The cerebral cortex of man, pp 109-134. New York: Macmillan.
Ploghaus A, Tracey I, Gati JS, Clare S, Menon RS, Matthews PM, Rawlins JN (1999) Dissociating pain from its anticipation in the human brain. Science 284:1979-1981.

Price DD, McHaffie JG, Larson MA (1989) Spatial summation of heatinduced pain: influence of stimulus area and spatial separation of stimuli on perceived pain sensation intensity and unpleasantness. J Neurophysiol 62:1270-1279.

Rainville P, Duncan GH, Price DD, Carrier B, Bushnell MC (1997) Pain affect encoded in human anterior cingulate but not somatosensory cortex. Science 277:968-971.

Robinson CJ, Burton H (1980) Somatic submodality distribution within the second somatosensory (SII), 7b, retroinsular, postauditory, and granular insular cortical areas of M. fascicularis. J Comp Neurol 192:93-108.

Rosen BR, Buckner RL, Dale AM (1998) Event-related functional MRI: past, present, and future. Proc Natl Acad Sci USA 95:773-780.

Sadato N, Ibanez V, Campbell G, Deiber MP, Le Bihan D, Hallett M (1997) Frequency-dependent changes of regional cerebral blood flow during finger movements: functional MRI compared to PET. J Cereb Blood Flow Metab 17:670-679.

Sikes RW, Vogt BA (1992) Nociceptive neurons in area 24 of rabbit cingulate cortex. J Neurophysiol 68:1720-1732.

Staub E, Tursky B, Schwartz GE (1971) Self-control and predictability: their effects on reactions to aversive stimulation. J Pers Soc Psychol 18:157-162.

Sutton S, Braren M, Zubin J, John ER (1965) Evoked-potential correlates of stimulus uncertainty. Science 150:1187-1188.

Svensson P, Beydoun A, Morrow TJ, Casey KL (1997) Human intramuscular and cutaneous pain: psychophysical comparisons. Exp Brain Res 114:390-392.

Talairach J, Tournoux P (1988) Co-planar stereotaxic atlas of the human brain. New York: Thieme.

Talbot JD, Marrett S, Evans AC, Meyer E, Bushnell MC, Duncan GH (1991) Multiple representations of pain in human cerebral cortex. Science 251:1355-1358.

Toma K, Honda M, Hanakawa T, Okada T, Fukuyama H, Ikeda A, Nishizawa S, Konishi J, Shibasaki H (1999) Activities of the primary and supplementary motor areas increase in preparation and execution of voluntary muscle relaxation: an event- related fMRI study. J Neurosci 19:3527-3534.

Towell AD, Purves AM, Boyd SG (1996) CO2 laser activation of nociceptive and non-nociceptive thermal afferents from hairy and glabrous skin. Pain 66:79-86.

Treede RD, Kenshalo DR, Gracely RH, Jones AK (1999) The cortical representation of pain. Pain 79:105-111.

Vogt BA, Sikes RW (2000) The medial pain system, cingulate cortex, and parallel processing of nociceptive information. Prog Brain Res 122:223-235.

Xu X, Fukuyama H, Yazawa S, Mima T, Hanakawa T, Magata Y, Kanda M, Fujiwara N, Shindo K, Nagamine T, Shibasaki H (1997) Functional localization of pain perception in the human brain studied by PET. NeuroReport 8:555-559. 